\title{
Assessing the Phytosanitary Risk Posed by an Intraspecific Invasion of Cryphonectria parasitica in Europe
}

\author{
Francesca Dennert, ${ }^{1, \dagger}$ Joana Beatrice Meyer, ${ }^{1,2}$ Daniel Rigling, ${ }^{1}$ and Simone Prospero ${ }^{1}$ \\ ${ }^{1}$ Swiss Federal Institute for Forest, Snow and Landscape Research WSL, CH-8903 Birmensdorf, Switzerland \\ ${ }^{2}$ Forest Protection and Forest Health Section, Federal Office for the Environment FOEN, CH-3003 Bern, Switzerland \\ Accepted for publication 13 August 2019.
}

ABSTRACT

\begin{abstract}
Intraspecific cryptic invasions may occur when new strains of an invasive species are introduced into an area where this species had already been introduced previously. In plant pathogens, such invasions are not well studied, even if, potentially, they can have severe consequences. Here, we investigated the effects of a potential intraspecific invasion in Europe of Cryphonectria parasitica, the causal agent of chestnut blight. Specifically, we tested the hypotheses that (i) non-European strains are more virulent on Castanea sativa than those already present in Europe because they have never encountered this new host, and (ii) the variation in virulence among strains is higher within native than within introduced populations. In a greenhouse, 2-year-old $C$. sativa seedlings were inoculated with Cryphonectria parasitica strains from South Korea, the United States, and Switzerland, and lesion development and seedling mortality were recorded weekly. Additionally, growth and sporulation of the strains
\end{abstract}

were measured in vitro on agar medium at 15 and $24^{\circ} \mathrm{C}$. Although lesion growth was similar for all strains, seedlings inoculated with strains from South Korea and Switzerland died faster than seedlings inoculated with strains from the United States. Moreover, in vitro strains from South Korea grew faster and produced more spores at both temperatures than the strains from the other two countries. In conclusion, our results did not support the two hypotheses. All strains, regardless of their origin, were found to be highly virulent on the inoculated chestnut seedlings. Nevertheless, current phytosanitary measures to avoid the introduction of new genotypes of C. parasitica into Europe should be further implemented.

Keywords: avirulence theory, Castanea spp., chestnut blight, cryptic invasion, disease control and pest management, ecology and epidemiology, mycology, virulence assay
Invasive plant pathogens represent a major threat for forest ecosystems because they can cause dramatic and persistent ecological changes (Ellison et al. 2005; Loo 2009). Well-known examples include white pine blister rust (Cronartium ribicola J. C. Fisch) in North America (Maloy 1997) and Dutch elm disease (Ophiostoma ulmi (Buisman) Nannf. and O. novo-ulmi Brasier) in North America and Europe (Gibbs 1978). Once an invasive pathogen has become established in a new area, its successful eradication is very difficult to achieve and complex containment measures have to be adopted (Picard et al. 2018). Hence, prevention of introduction and establishment (proactive capacity) (Early et al. 2016), although difficult to implement, is the best way to combat invasive pathogens. This applies not only to new species but also to additional genotypes of already introduced species (Schrader and Unger 2003).

In most biological invasions, only a small fraction of the genotypic and phenotypic diversity of a source population is, at least initially, introduced in a new area, leading to a demographic bottleneck. For example, the epidemic of ash dieback caused by Hymenoscyphus fraxineus (T. Kowalski) Baral, Queloz \& Hosoya in Europe probably resulted from the introduction of only two fungal genotypes (Gross et al. 2014; Schoebel et al. 2017). Similarly, one or two genotypes of the ambrosia fungus Raffaelea lauricola T. C. Harr, Fraedrich \& Aghayeva, possibly introduced from Asia, are responsible for the invasive laurel wilt disease in the southeastern United States (Wuest et al. 2017). However, even when

${ }^{\dagger}$ Corresponding author: F. Dennert; francesca.dennert@wsl.ch

*The $\boldsymbol{e}$-Xtra logo stands for "electronic extra" and indicates that one supplementary table is published online.

The author(s) declare no conflict of interest.

(c) 2019 The American Phytopathological Society an invasive pathogen has already become established, invasions by new genotypes (so-called intraspecific cryptic invasions) (Morais and Reichard 2018) can potentially have significant consequences. First, the new genotypes may modify the population biology of the species in the introduced range; for example, by allowing or increasing sexual reproduction. This was the case when the A2 mating type of the potato late blight pathogen Phytophthora infestans (Mont.) de Bary was introduced into Europe (Hohl and Iselin 1984; Mariette et al. 2016). Although, in some European regions, clonal populations remained dominant, in other regions, mating type frequencies rapidly changed, and the pathogen started to reproduce sexually (Kiiker et al. 2018; Montarry et al. 2010). Sexual reproduction itself has the potential to generate new genotypes, which may be better adapted to local conditions than the old ones. Second, newly introduced genotypes may be more virulent toward the new host than the previously introduced ones, especially when a time lag exists between introduction events. According to the "avirulent hypothesis", parasites should coevolve with their hosts to become avirulent in order to avoid extinction of the host and of themselves (May and Anderson 1983). However, because some degree of virulence is unavoidable for pathogen reproduction and transmission (transmission-virulence trade-off), coevolution will most likely not result in avirulent genotypes but, rather, in genotypes with an intermediate virulence to maximize their transmission and, at the same time, avoid host extinction (Alizon et al. 2009). Therefore, we may expect that genotypes from old introductions are better adapted (i.e., less virulent) to the new host than newly introduced genotypes, which could cause increased damage.

In this study, using greenhouse and in vitro inoculation experiments, we assessed the risk represented by invasion of new strains of the chestnut blight fungus Cryphonectria parasitica in Europe. C. parasitica infects chestnut (Castanea spp.) trees through wounds in the bark and induces the formation of necrotic lesions (so-called cankers) that may girdle and eventually kill the affected 
tree part (Rigling and Prospero 2018). In its native range in Asia (China, Japan, and Korea), the fungus is a weak pathogen on native chestnut species (Castanea mollissima Blume and $C$. crenata Siebold \& Zucc.). In the 20th century, it was accidentally introduced first into North America and then into Europe, causing severe damage on American (C. dentata (Marsh.) Borkh.) and European (C. sativa Mill.) chestnut, respectively. Previous studies (Dutech et al. 2012; Liu and Milgroom 2007) showed that, as expected, genetic diversity of Cryphonectria parasitica is higher in Asia than in North America and Europe, which likely is also reflected in variation of phenotypic traits, including virulence.

Chestnut blight was first detected in Europe in 1938 in northern Italy (Biraghi 1946) and, today, it is present in most European chestnut-growing areas (Robin and Heiniger 2001). Because chestnut blight is currently widespread in continental Europe and its eradication is not realistic anymore, $C$. parasitica has recently been recommended for listing as a "regulated non-quarantine pest" by the European Food Safety Authority (EFSA Panel on Plant Health 2016). Population genetic studies showed that the pathogen was introduced to Europe several times (Dutech et al. 2010, 2012). One introduction occurred from the United States, from where the fungus was accidentally introduced to Italy (Anagnostakis 1987). Another introduction directly from Asia targeted southwestern France. Although, in the United States, chestnut blight virtually eliminated the native American chestnut (Shackleton et al. 2018), in Europe, the epidemics followed a milder course thanks to the appearance and spontaneous spread of a mycovirus ( $\mathrm{CHV}-1)$ that reduces virulence and sporulation of infected $C$. parasitica strains

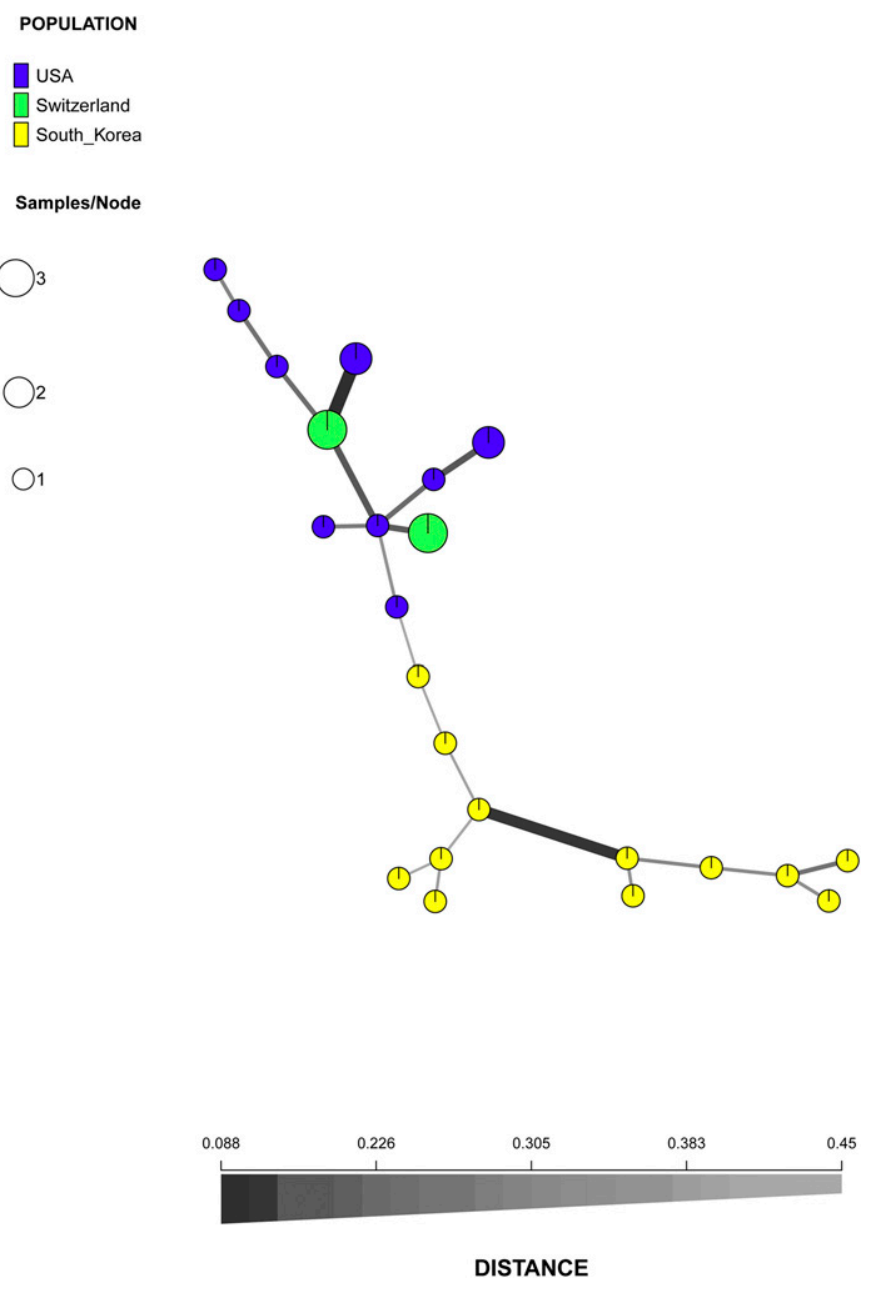

Fig. 1. Minimum spanning network based on Bruvo's distances using microsatellite data (Prospero and Rigling 2012) showing the genetic relationships among the 29 Cryphonectria parasitica strains used in the study.
(Grente 1965; Heiniger and Rigling 1994). Additionally, the European chestnut is slightly less susceptible to $C$. parasitica than the American chestnut; however, it is unclear how much this influenced the $C$. parasitica epidemic in Europe.

New introductions of $C$. parasitica to Europe in recent years are not known. Most likely this was prevented by the implementation of international and national phytosanitary measures (Eschen et al. 2015). However, for several reasons (e.g., latent infections with no expression of symptoms) (Leclerc et al. 2014), prevention and control policies might fail, resulting in new introductions. The aim of this study was to understand how an accidental introduction to Europe of $C$. parasitica genotypes from other continents would affect the European population of Castanea sativa. Specifically, we tested the following two hypotheses: (i) strains from South Korea and North America are more virulent on $C$. sativa than those already present in Europe because they have never encountered this new host and (ii) the variation in virulence among strains is higher within native (South Korea) than within introduced (North America and Switzerland) Cryphonectria parasitica populations. Here, we define virulence as a quantitative trait (i.e., the degree of damage caused by a pathogen to a specific host) (Sacristàn and Garcì-Arenal 2008). For testing the two hypotheses mentioned above, we inoculated $C$. parasitica strains from South Korea, the United States, and Switzerland on 2-year-old European chestnut seedlings and regularly assessed lesion development and mortality. Because not only virulence but also other biological traits (e.g., host range, sporulation and dispersal capacity, and growth-temperature interactions) determine the invasiveness of a pathogen (Philibert et al. 2011), at the same time we assessed growth and sporulation of the selected $C$. parasitica strains in vitro at two different temperatures. We then tested whether in vitro characteristics are linked to in planta virulence of the $C$. parasitica strains.

\section{MATERIALS AND METHODS}

C. parasitica strains. In total, 29 C. parasitica strains from one native (South Korea) and two introduced (United States and Switzerland) populations were selected for the study (Supplementary Table S1; Fig. 1). The 12 South Korean strains were recovered at eight different locations from infected Castanea crenata trees and, based on microsatellite (simple-sequence repeat [SSR]) analysis (Prospero and Rigling 2012), they represented 12 different genotypes. The 11 strains from the United States (nine different SSR genotypes) were collected at five sites in Maryland, New Hampshire, West Virginia, New York State, and Kentucky from $C$. dentata trees. Finally, the six Swiss strains were isolated at four different locations in southern Switzerland (Ticino) from bark cankers on $C$. sativa trees. These strains were selected to represent the two most widespread and, thus, most likely successful invasive genotypes in that part of Switzerland (CpMG33 and CpMG15) (Prospero and Rigling 2012). The genetic relationships among the 29 selected strains were visualized by producing a minimumspanning network (MSN) based on Bruvo's distances calculated with the SSR data (Prospero and Rigling 2012). For this analysis, the function "bruvo.msn" of the R package "poppr" (Kamvar et al. 2014) was used. Genetic diversity of the Cryphonectria parasitica populations was assessed by calculating allelic richness per SSR locus standardized by sample size using the function "allel.rich" of the R package "PopGenReport" (Adamack and Gruber 2014). Because Swiss and North American strains grouped together in the MSN (see Results), for this analysis, they were considered to belong to the same population.

Before inoculation, the absence of a CHV-1 infection in all $C$. parasitica strains was verified based on culture morphology and molecular analysis. Culture morphology on potato dextrose agar (PDA) (39 g/liter; Difco) was assessed after incubating the plates in 
the dark at room temperature for 7 days followed by an additional incubation under daylight for another 7 days at room temperature (Bissegger et al. 1997). After this treatment, virus-free strains typically showed a characteristic orange morphology. For molecular analysis, strains were grown on cellophane PDA and, after 7 days, the mycelium was harvested and lyophilized (Hoegger et al. 2000). Total RNA was extracted with the RNEasy Plant mini kit (Qiagen) as described in the manufacturer's protocol. cDNA was obtained with the Maxima first-strand cDNA synthesis kit (Thermo Fisher Scientific) and used to amplify a specific region of the open reading frame (ORF)-A from $\mathrm{CHV}-1$ with the method described by Gobbin et al. (2003). The absence of the ORF-A fragment was then confirmed by electrophoresis on $1.5 \%$ agarose gel.

Castanea sativa population. The virulence of the selected C. parasitica strains was assessed on 2-year-old Castanea sativa seedlings (stem diameter 10 to $20 \mathrm{~mm}$ ) issued from a wild $C$. sativa population from southern Germany. Three months before inoculation, the bare-root seedlings were planted in plastic pots containing a soil substratum composed of bark compost, peat, wood fibers, and 14\% mineral material, placed in the forest nursery of the Swiss Federal Research Institute WSL, and watered as required.

Virulence assay. The virulence assay was performed between July and October 2017 in the biosafety greenhouse facility at WSL with the Cryphonectria parasitica and Castanea sativa populations described above. Inoculations were conducted as described by Hoegger et al. (2002). Briefly, the $C$. sativa seedlings were inoculated into the stem with an agar plug of Cryphonectria parasitica culture grown on PDA for 7 days at room temperature. A hole of $5 \mathrm{~mm}$ in diameter was cut into the bark with a cork borer and filled with a PDA plug of a $C$. parasitica culture. The plug was placed in the wound with the mycelium side facing the cambium and then covered with tape to prevent desiccation. Control seedlings were inoculated with sterile PDA plugs. For each $C$. parasitica strain, five chestnut seedlings were inoculated. The experiment was performed at room temperature and under natural light. Seedlings were watered as needed (one to two times per week) to keep the soil always moist. The pots containing the seedlings were randomly distributed on nine greenhouse trolleys which were moved around weekly to avoid any influence of their position on the experiment. As proxies for virulence, lesion size and seedling mortality were recorded. Starting at 2 weeks after inoculation, lesion length and width were measured using a millimeter ruler once per week until 23 weeks after inoculation, when all seedlings except four and the controls had died (see Results). Mortality was defined as time until seedling death (wilting of the foliage above the inoculation point) and was recorded in weeks.

Reisolation of $\boldsymbol{C}$. parasitica. To verify that the dead seedlings were effectively killed by the inoculated $C$. parasitica strain, the bark at the edge of the lesion was removed with a knife and the presence of the characteristic pale-brown mycelial fans (Rigling and Prospero 2018) was checked. Moreover, 15 dead seedlings were randomly selected and $C$. parasitica was reisolated from the lesions. For this, small pieces of bark and mycelium from the lesion were plated on $1.5 \%$ water agar (Industrial agar number 2; Pronadisa) and plates were incubated for 7 days at room temperature in the dark. After this period, growing $C$. parasitica colonies were transferred to PDA and incubated for 7 days at room temperature in the dark. A mycelial plug of the reisolated culture was then paired on PDA with a mycelial plug of the original strain. The plates were successively incubated in the dark at room temperature for 7 days. A merging reaction between the two colonies (Bissegger et al. 1997) confirmed that the reisolated strain corresponded to the inoculated one.

Growth and sporulation in vitro. The growth of the 29 studied $C$. parasitica strains was measured in vitro at two different temperatures $\left(15\right.$ and $24^{\circ} \mathrm{C}$ ) under a light intensity of 3,330 lx and a 14-h photoperiod. Mycelial agar plugs of $5 \mathrm{~mm}$ in diameter originating from the growing margin of pure cultures were placed upside down on the center of PDA plates $(8 \mathrm{~cm}$ in diameter). Size of the colony was measured with a millimeter ruler daily for 7 days (after this period, the majority of colonies completely colonized the Petri plate). Orthogonal lines were traced on the Petri plate and colony growth was measured along the lines. The two measurements corresponded approximately to the major and minor axis of an ellipsis and were used to calculate the colony size.

The colonies were then incubated under the same conditions for 7 more days, after which the sporulation was assessed. At this time, all C. parasitica cultures completely covered the Petri plate. Spores were washed from the surface of the colonies with $5 \mathrm{ml}$ of sterile distilled water and then quantified using a hemocytometer $(0.1-\mathrm{mm}$ depth, $0.0025 \mathrm{~mm}^{2}$ ) (Neubauer) under a light microscope at $\times 40$ magnification.

Data analysis. Data were analyzed with the statistical software $\mathrm{R}$, version 3.4.1 (R Core Team 2014). In the greenhouse assay, the five seedlings inoculated with the same $C$. parasitica strain were considered to be biological replicates. At the first measurement, 2 weeks after inoculation, lesion width significantly positively correlated with seedlings' diameter (Spearman's correlation, $\rho=0.308, P=7.224 \times 10^{-05}$ ); therefore, only lesion length data were used for statistical analysis. Lesion length data were analyzed until the first seedlings died at 7 weeks

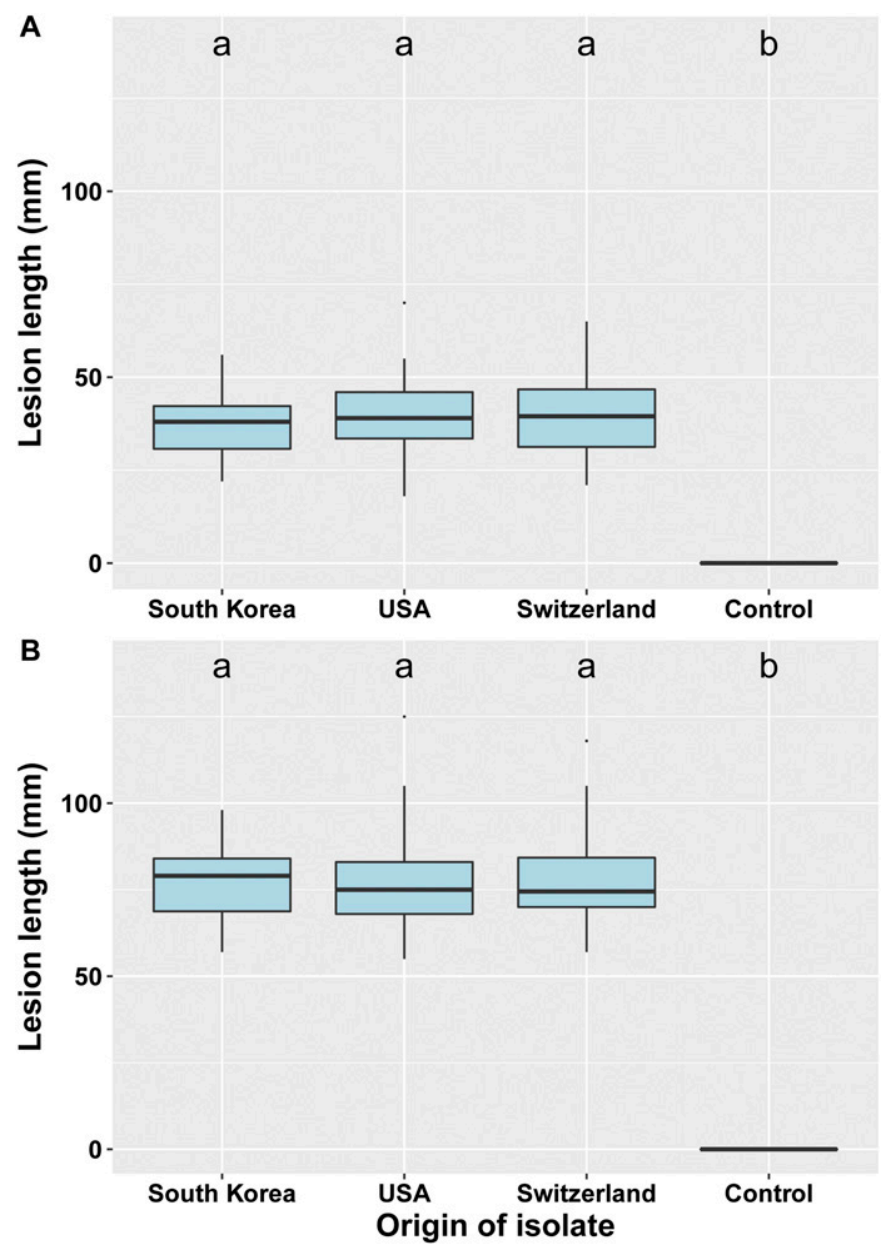

Fig. 2. Box plots of the length of lesions (in millimeters) caused by Cryphonectria parasitica on 2-year-old Castanea sativa seedlings inoculated with strains from South Korea (12 isolates), United States (11 isolates), and Switzerland (6 isolates) A, 2 weeks (first measurement) and B, 7 weeks (last measurement) after inoculation. Black lines in the boxes show medians. Letters above the graph indicate significant differences in the lesion length between strains from different countries (nested linear model followed by Tukey's posthoc test, significance level $=0.05)$. 
postinoculation. The time until seedling mortality was recorded in weeks after inoculation, and differences between country of origin of the strains were inferred with a linear model, where the strain was nested within the country, followed by a Tukey posthoc test (significance threshold: $P<0.05$ ). The stem diameter of the seedlings was considered to be a confounding factor in the model. Differences in variances between the populations were analyzed with Bartlett's test. A survival analysis was performed by plotting Kaplan-Meier survival curves with the function "ggsurvplot" of the package "survminer", and comparing them with the log-rank test.

In the in vitro experiment, differences in growth between strains from different countries were inferred 7 days postinoculation, while differences in sporulation 14 days postinoculation were inferred as described above for the greenhouse experiment. Correlations at the isolate level between all analyzed parameters (mortality, lesion length, in vitro colony size, and in vitro sporulation) were shown in a heat map. Spearman's correlation analysis was used (significance level: $P<0.05$ ) because of the nonnormal distribution of the data and the robustness to outliers.

\section{RESULTS}

The MSN showed that the Swiss $C$. parasitica strains used in this study clearly grouped with the strains originating from the United States, whereas the South Korean strains were located on another sector of the network (Fig. 1). Genetic diversity estimated by the allelic richness across loci was considerably higher in the South Korean population (4.6) than in the combined Swiss and North American populations (2.5).

Virulence assay. Five seedlings were inoculated with each C. parasitica strain and the size of the lesions was measured weekly until 7 weeks after inoculation. Lesion length 2 and 7 weeks after inoculation was not significantly different between strains from different countries (Fig. 2). There were also no significant differences in variation of lesion length between countries.

The first seedlings died 7 weeks after inoculation (Fig. 3), and the median mortalities (i.e., when $50 \%$ of the seedlings had died) were 11 weeks postinoculation (strains from South Korea), 12 weeks postinoculation (strains from the United States), and 10 weeks postinoculation (strains from Switzerland). The highest variation in time until death was in seedlings inoculated

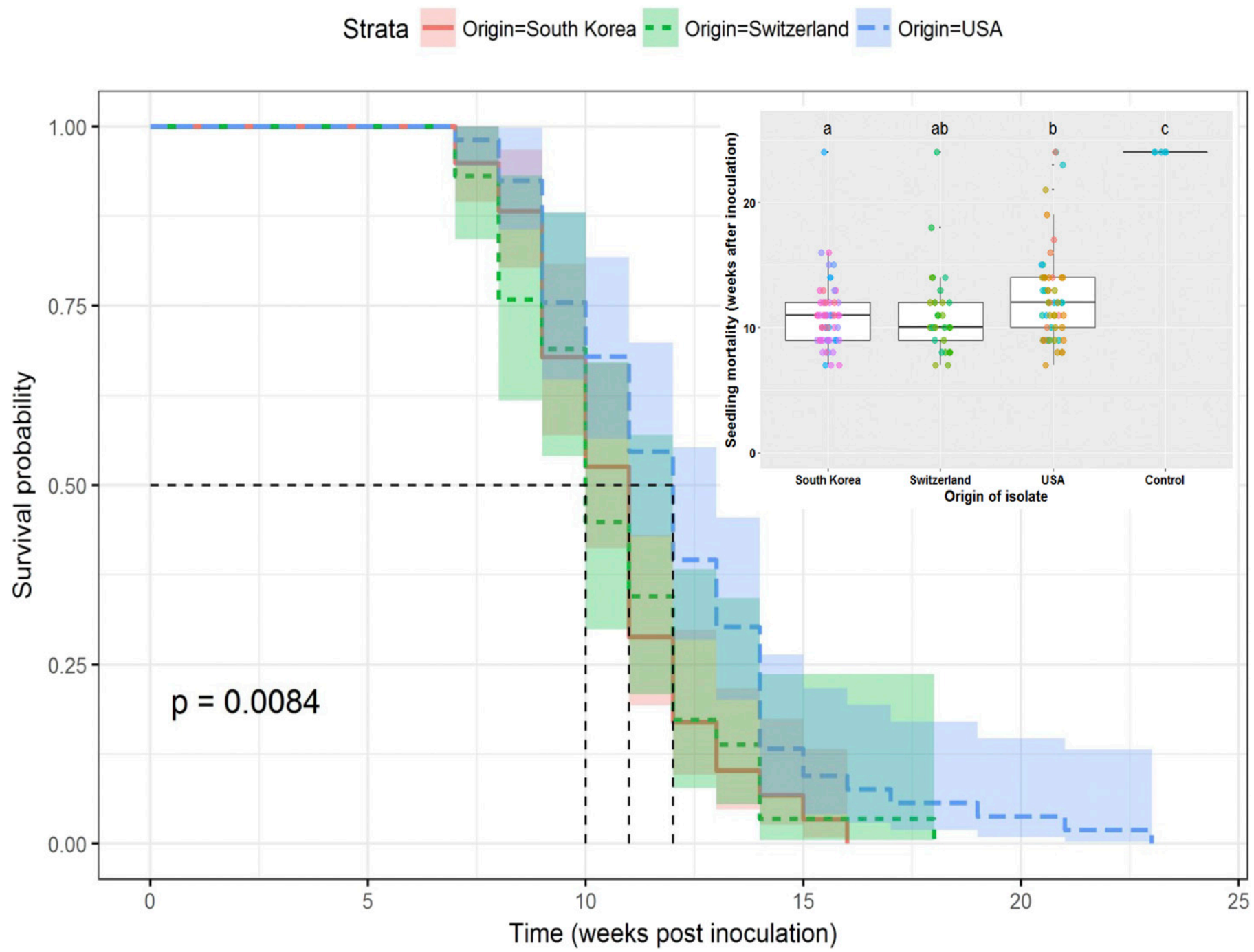

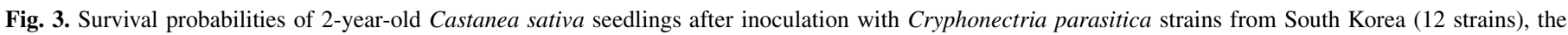

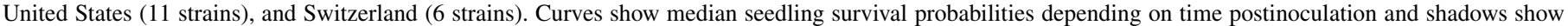

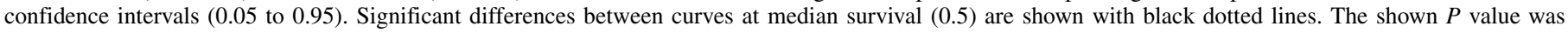

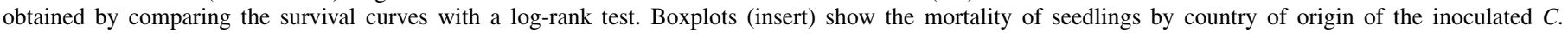

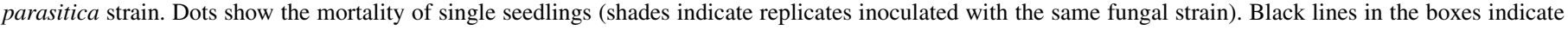

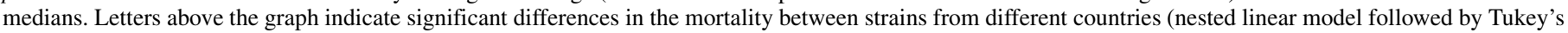

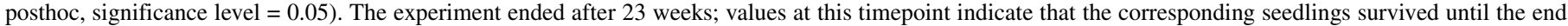
of the experiment. 
with strains from the United States (standard deviation = 3.8 weeks), while the smallest was in seedlings inoculated with strains from South Korea (standard deviation $=2.7$ weeks). The variation of time until seedling death was significantly different between countries (Bartlett's test, $P=2.2 \times 10^{-16}$ ). Overall, the time until the seedlings died was significantly shorter in seedlings inoculated with strains from South Korea compared with strains from the United States but there was no significant difference between strains from South Korea and Switzerland (Fig. 2). Two strains from South Korea (SKo77 and SKo178) (Fig. 4) killed all inoculated seedlings within 10 weeks after inoculation. Four seedlings, each inoculated with a different C. parasitica strain (Sko134 from South Korea, MD-1-2 and BRU1 from the United States, and M3963 from Switzerland) (Fig. 4) survived until the end of the experiment at 23 weeks after inoculation. Control seedlings inoculated with sterile PDA plugs did not develop any lesion and survived without symptoms until the end of the experiment. Visual inspection of bark lesions that developed on dead seedlings revealed the presence of the typical mycelial fans of $C$. parasitica under the bark. Reisolations and pairing tests showed that the $C$. parasitica strain present in the lesions was the one originally inoculated.

Growth and sporulation in vitro. The $C$. parasitica strains used for the inoculation assay were also grown in vitro at 15 and $24^{\circ} \mathrm{C}$ and colony growth and sporulation were assessed. Colony size after 7 days at $15^{\circ} \mathrm{C}$ (Fig. 5A) was significantly larger in strains from South Korea compared with strains from the United States and Switzerland. Variation in colony size was highest in strains from South Korea, followed by strains from the United States and from Switzerland. At $24^{\circ} \mathrm{C}$, similar results were obtained (Fig. 5B). In vitro growth at both tested temperatures was significantly positively correlated (Spearman's $\rho=0.55, P=$ 0.002) (Figs. 6 and 7). Although the ranking of strains for colony size was different at the two tested temperatures, these differences were not dependent on the geographic origin of the isolates (Fig. 6).

Sporulation was assessed after 14 days by washing the spores from the plate with sterile distilled water and counting the spores in the suspensions. The median number of spores per plate at $15^{\circ} \mathrm{C}$ (Fig. 5C) was slightly higher for strains from South Korea compared with strains from the United States and Switzerland but differences between countries were not significant. At $24^{\circ} \mathrm{C}$ (Fig. 5D), sporulation was significantly different for strains from different countries, with strains from South Korea producing the highest number of spores per plate, followed by Swiss and American strains. The variation within populations was again highest in the South Korean strains. Overall, strains from South Korea grew faster at both tested temperatures and produced more spores per plate than strains from the United States and Switzerland. Moreover, South Korean strains showed a greater variation within the population for colony growth and sporulation compared with the two other studied populations.

Correlations between in vivo and in vitro data. Spearman's correlations between all the assessed parameters were inferred and shown in a heat map (Fig. 6). Lesion length and seedling mortality were significantly negatively correlated $(\rho=$ $-0.42, P=0.01$ ) (i.e., larger lesions resulted in a faster seedling death). In the in vitro experiment, significant correlations $(P<$ $0.05)$ were found between colony size (i.e., faster growth in vitro) and sporulation at both assessed temperatures. The correlations between parameters from the inoculation assay and from the in vitro experiment were not significant. Lesion length on seedlings showed no clear positive or negative correlation with colony growth or sporulation in the in vitro experiment ( $\rho$ approximately 0 ). Similarly, although seedling mortality was weakly negatively correlated with in vitro growth and sporulation at $24^{\circ} \mathrm{C}$, correlations were not significant (for colony area, $\rho=-0.3, P=0.09)$.
South Korea

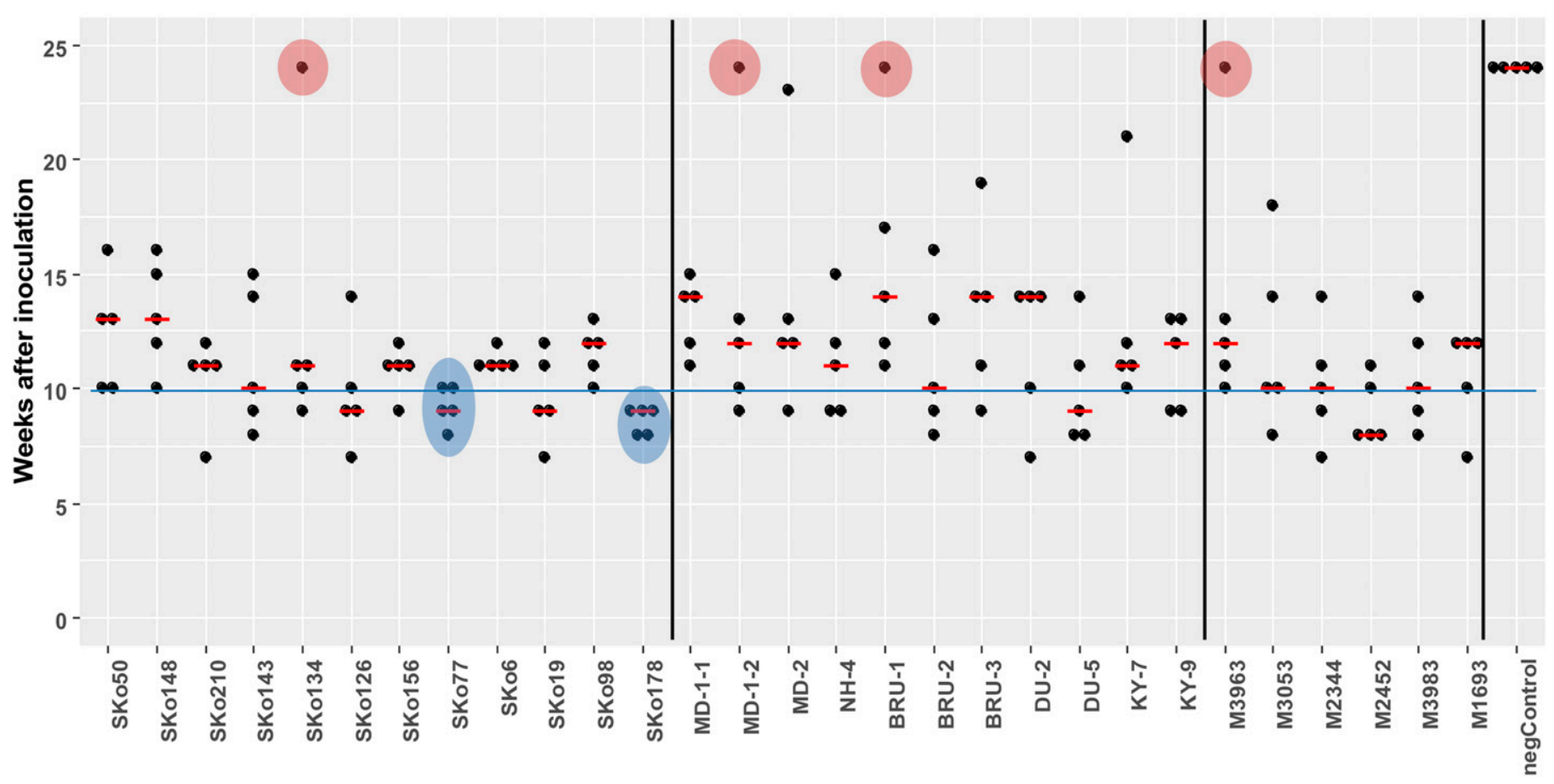

Isolate

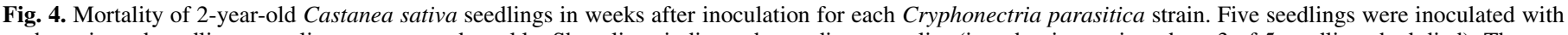

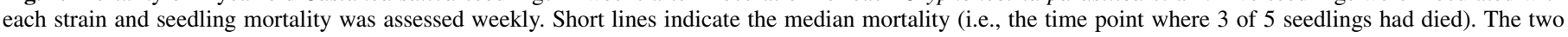

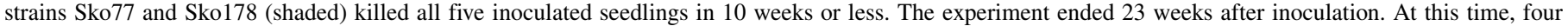
seedlings were still alive, all inoculated with different strains. 


\section{DISCUSSION}

In this study, we assessed the risk posed by an intraspecific invasion of $C$. parasitica from South Korea and North America to Europe by performing in planta and in vitro inoculation experiments. All $C$. parasitica strains regardless of their population of origin, were found to be highly virulent, producing large bark lesions and high mortality of the inoculated chestnut seedlings. Mortality (in weeks postinoculation) was only affected by seedling diameter (i.e., seedlings with smaller diameters died faster). A longer survival of seedlings with a larger diameter was previously reported in two studies in which $C$. parasitica strains were inoculated onto Castanea sativa, C. crenata, and C. sativa $\times$ C. crenata seedlings (Pažitný et al. 2018; Xenopoulos and Papachatzis 1999). Our results suggest that an intraspecific invasion of $C$. parasitica from North America and South Korea would not necessarily lead to an increased mortality of $C$. sativa in Europe. As known from the invasion history of chestnut blight (Dutech et al. 2012) and confirmed by our MSN, the Swiss Cryphonectria parasitica population is more closely related to the North American than to the South Korean population. Therefore, we would have rather expected the Swiss strains to behave more as the North American ones did.

Although the number of strains tested in our study is most likely not sufficient to infer sound conclusions about the evolution of European C. parasitica populations, our findings do not seem to support the avirulence theory (May and Anderson 1983). Based on this theory, the $C$. parasitica population in southern Switzerland, after its establishment in the 1940s, should have evolved toward lower virulence to avoid host extinction. If we consider roughly one fungal generation per year (i.e., one sexual reproduction event per year), the coevolution time between the parasite ( $C$. parasitica $)$ and the host (Castanea sativa) would be approximately 80 fungal generations, eventually still too short to allow the selection for less virulent genotypes. However, other particularities of the European pathosystem could have influenced the evolution of the hostpathogen interaction. One is the presence of the hypovirus, which reduces the virulence of the infected isolates and, therefore, could counterbalance the selection pressure for less virulent genotypes in the Cryphonectria parasitica population. In North American populations, on the other hand, hypovirulence could not become established and strains with a reduced virulence may have effectively been selected to avoid the extinction of the local host (Castanea dentata). This process may have been accelerated by the particularly high susceptibility of $C$. dentata to Cryphonectria parasitica. Even without the effect of hypovirulence, host extinction in Europe would most likely have taken a longer time than in the United States because Castanea sativa trees usually support multiple infections before they die. Cryphonectria parasitica is native to South Korea and, in accordance with the avirulence theory, a weak pathogen on the local chestnut species (Anagnostakis 1992). The greater genetic diversity of South Korean strains did not lead to a greater variation in lesion length among these strains compared with the strains from the two invasive

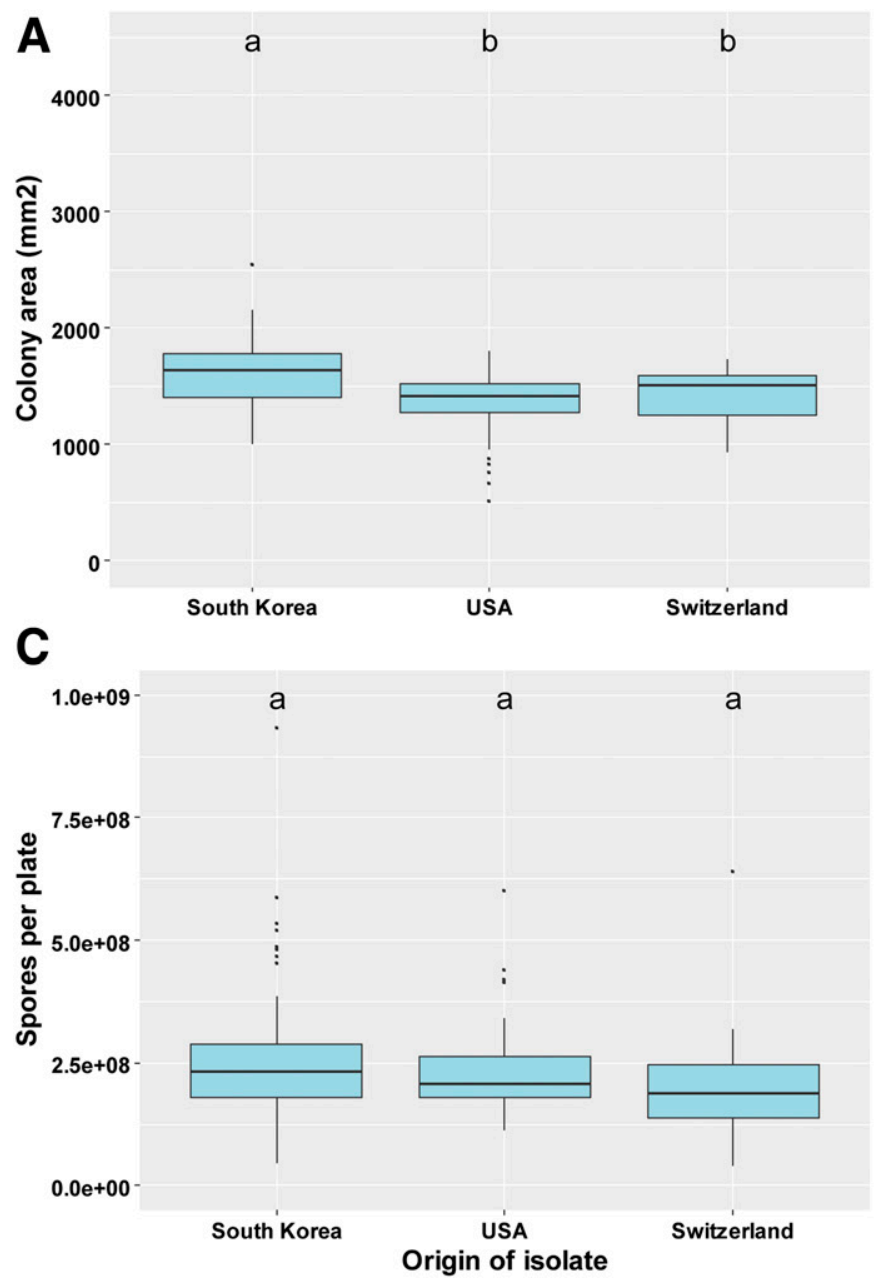

\section{B \\ D}
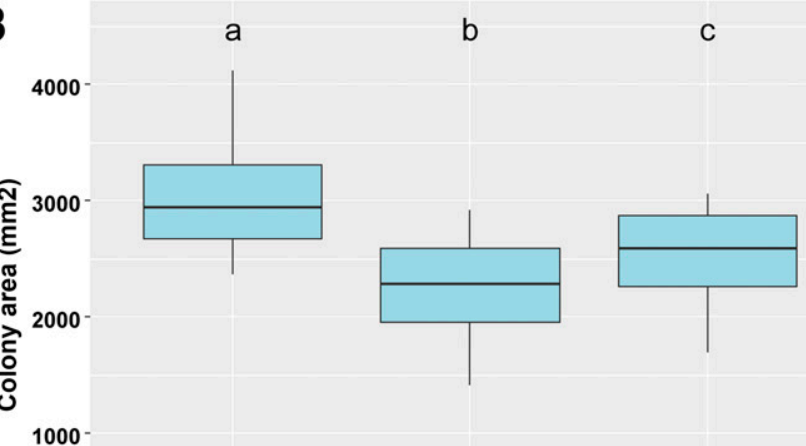

1000

0
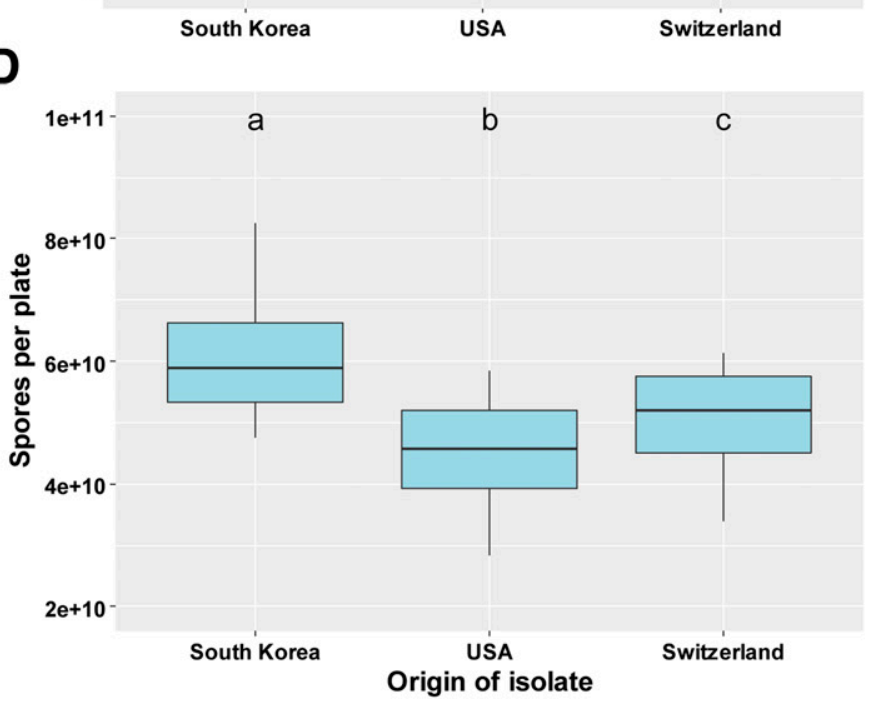

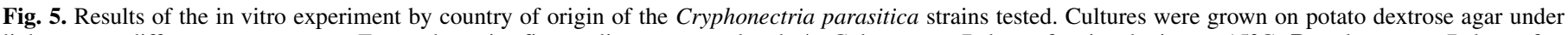

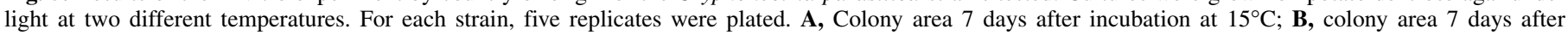

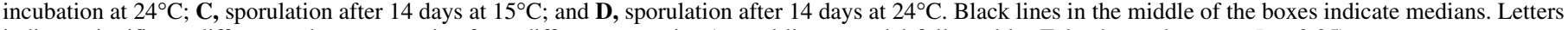
indicate significant differences between strains from different countries (nested linear model followed by Tukey's posthoc test, $P<0.05$ ). 
populations. Therefore, we could not confirm the hypothesis that a more variable natural population exhibits greater variability in virulence. These results suggest that high virulence on European chestnut is a general phenotypic trait of $C$. parasitica from South Korea and also, presumably, from other areas in Asia. Following this line, the mechanism behind virulence is likely associated with important fitness traits of the fungus in its native range and, therefore, under strong selection.

In our inoculation study, we only found minor differences in virulence among $C$. parasitica strains from native and introduced populations. In addition to the relatively low number of strains considered, the specific inoculation technique applied might have accounted for this result. First, inoculating a mycelial plug in a wound previously made in the bark of a seedling only allows assessment of one aspect of virulence (i.e., parasitic mycelial growth in the bark). Second, the method is very effective (i.e., always results in a lesion) but is far from the natural infection process. In nature, an infection is caused by sexual or asexual spores which germinate in fresh wounds (e.g., growth cracks) present in the bark tissue (Rigling and Prospero 2018). Because the inoculum pressure is much lower than in the inoculation experiment, differences in virulence among genotypes may be more evident. Unfortunately, spore inoculations are experimentally more difficult to perform than inoculations with mycelial plugs.
The seedling inoculation trial that we conducted has proven to be very useful for predicting the outcome of potential intraspecific invasions in $C$. parasitica. Nevertheless, such experiments are work and time intensive, and require living plant material and appropriate greenhouses to fulfill all biosafety requirements. In vitro studies without living plant material, conducted under conditions that reflect the environment where the invasion would take place, may represent a valuable alternative or provide complementary information. Here, we assessed, at two different temperatures, two biological traits which may potentially also affect invasiveness of the tested C. parasitica strains (i.e., growth and sporulation). All strains, disregarding the population of origin, showed a larger colony area after incubation at 24 than at $15^{\circ} \mathrm{C}$, which is in line with the results of previous studies (Bazzigher 1981; Bryner and Rigling 2011). However, individual strains appeared to be better adapted to one of the tested temperatures, although this was not linked to their geographic origin. The potential importance of temperature for the selection of specific $C$. parasitica strains, thereby influencing the course of the chestnut blight epidemics, was previously shown by Bryner and Rigling (2011).

Because C. parasitica strains from South Korea grew faster and sporulated more in vitro than those from the other two populations at both tested temperatures, one could imagine a higher virulence of South Korean strains. However, no

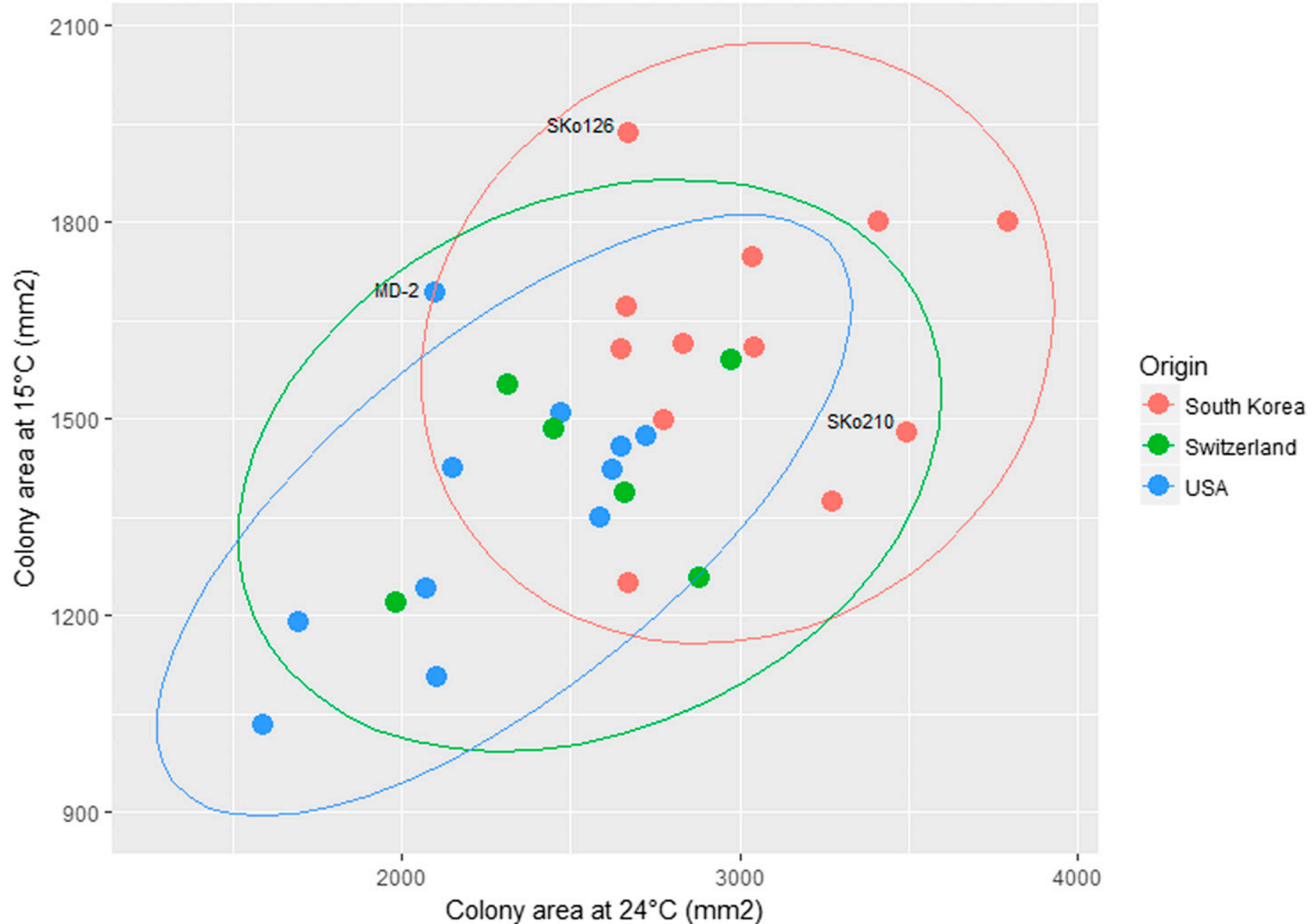

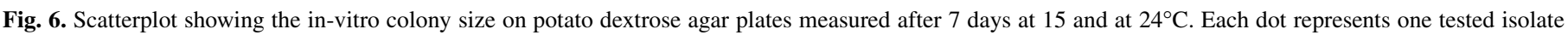

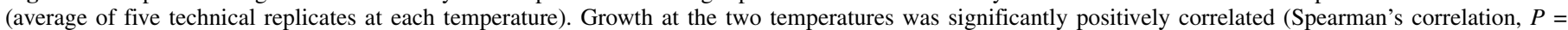

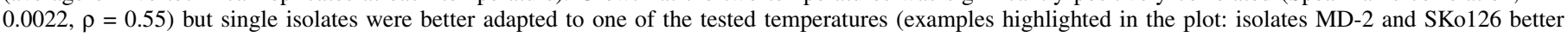

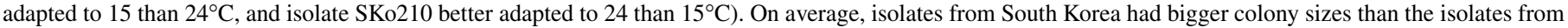
the other two countries at both tested temperatures (upper-right ellipse). 
significant correlation was found between colony growth and sporulation measured in vitro and the virulence toward the seedlings at the single isolate level. Similarly, although variability in colony growth and sporulation on PDA within South Korean isolates was higher than within Swiss and U.S. isolates, this was not observed in the seedling inoculation experiment. Hence, our results suggest that in vitro experiments can be used in a limited way to predict the outcome of in planta inoculation experiments. However, they can represent a valuable approach to assess biological traits that are difficult to measure on living plant material (e.g., sporulation). In our specific case, the observed variation in sporulation among $C$. parasitica strains on PDA may yet indicate differences in invasiveness. Even though all tested strains are highly virulent toward chestnut seedlings, only those with a good sporulation capacity may be able to rapidly spread and become successful invaders. Thus, a combination of in vitro and in planta experiments may allow for a more precise assessment of phytosanitary risks.

In conclusion, our study suggests that an introduction of C. parasitica strains from South Korea and the United States to Europe would not necessarily result in an increased mortality of
Castanea sativa. However, to definitively confirm this result, strains from additional populations (e.g., China and Japan) should also be tested on different provenances of European chestnut trees. Because Robin et al. (2017) showed a thermal adaptation in Cryphonectria parasitica during range expansion, it would be particularly important to consider non-European strains from all climates where the pathogen occurs. Similarly, testing more strains from Europe would allow a better accounting of eventual differences in the European population due to the different source populations (Asia and North America). Although the non-European C. parasitica strains seem not to be more virulent than the ones already present in Europe, other problems could arise with their introduction. In particular, an invasion by new strains could result in an increase of the vegetative compatibility (vc) type diversity, which could negatively affect spread of the hypovirus responsible for hypovirulence. In fact, high vc type diversity is considered to be one of the main reasons for the failure of biocontrol of chestnut blight with hypovirulence in North America (Milgroom and Cortesi 2004). Thus, it is mandatory that current phytosanitary measures to avoid the introduction of new C. parasitica strains in Europe are further implemented.

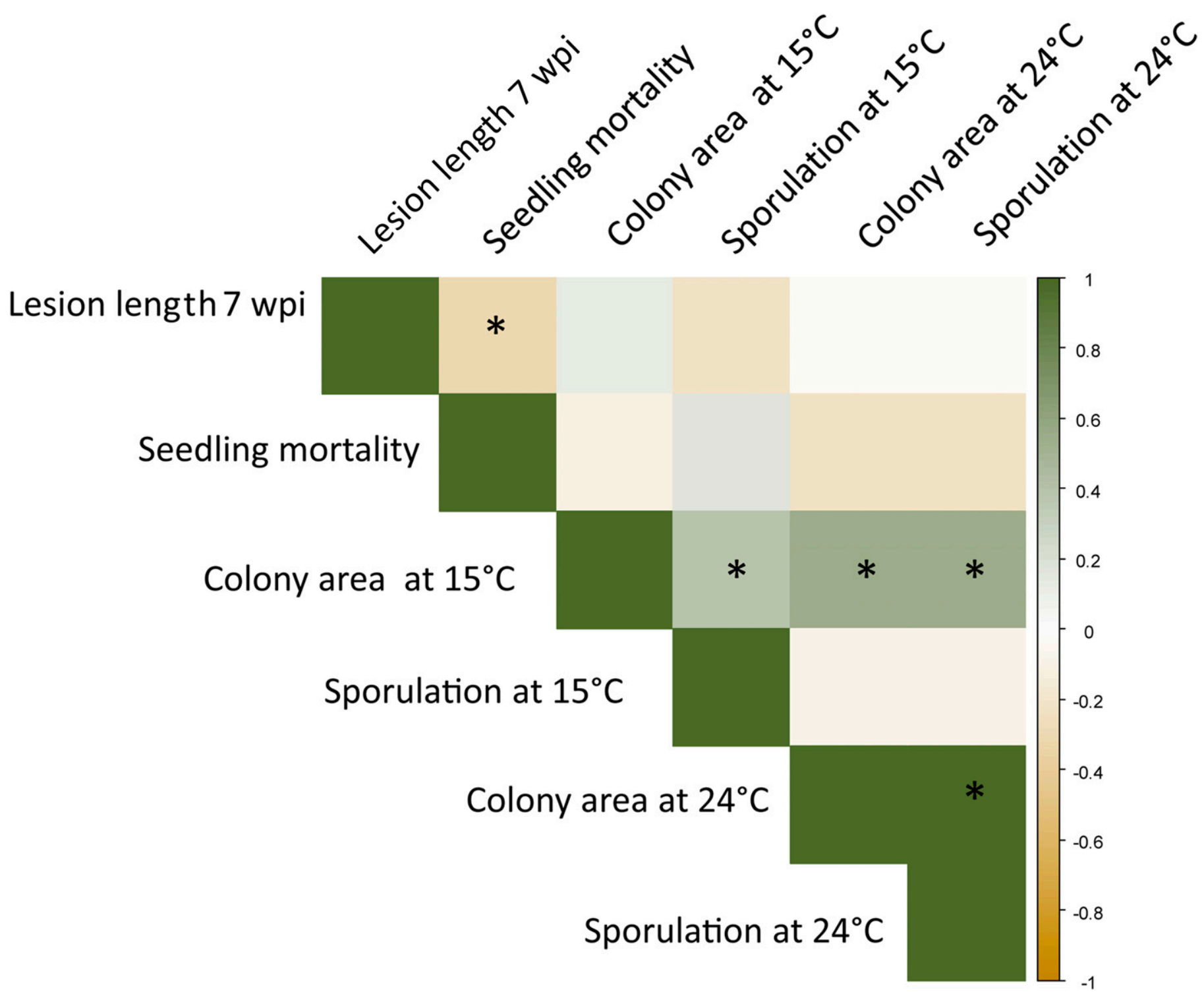

Fig. 7. Heat map showing Spearman's correlations between data from the in vivo greenhouse experiment and in vitro experiments. Significant correlations are indicated with asterisks $(P<0.05)$. The bar on the right shows the correlation coefficient (Spearman's $\rho)$. Lesion length 7 wpi indicates the length of the lesions measured 7 weeks after seedling inoculation with Cryphonectria parasitica strains. Seedling mortality is expressed as number of weeks until the inoculated seedlings died. Colony areas at 15 and $24^{\circ} \mathrm{C}$ are the colony sizes measured 7 days after inoculation on potato dextrose agar (PDA) plates. Sporulation at 15 and at $24^{\circ} \mathrm{C}$ indicates the number of spores produced by a strain on a PDA plate 14 days after inoculation. 


\section{ACKNOWLEDGMENTS}

We thank H. Blauenstein for help with the greenhouse experiment; L. Stauber for help with figure plotting; S. H. Lee, J.-H. Park, and M. Milgroom for providing the $C$. parasitica strains from South Korea and the United States; and two anonymous reviewers for helpful comments on the manuscript.

\section{LITERATURE CITED}

Adamack, A. T., and Gruber, B. 2014. PopGenReport: Simplifying basic population genetic analyses in R. Methods Ecol. Evol. 5:384-387.

Alizon, S., Hurford, A., Mideo, N., and Van Baalen, M. 2009. Virulence evolution and the trade-off hypothesis: History, current state of affairs and the future. J. Evol. Biol. 22:245-259.

Anagnostakis, S. L. 1987. Chestnut blight: The classical problem of an introduced pathogen. Mycologia 79:23-37.

Anagnostakis, S. L. 1992. Measuring resistance of chestnut trees to chestnut blight. Can. J. For. Res. 22:568-571.

Bazzigher, G. 1981. Selection of blight-resistant chestnut trees in Switzerland. Eur. J. For. Pathol. 11:199-207.

Biraghi, A. 1946. Il cancro del castagno causato da Endothia parasitica. Ital. Agric. 7:1-9.

Bissegger, M., Rigling, D., and Heiniger, U. 1997. Population structure and disease development of Cryphonectria parasitica in European chestnut forests in the presence of natural hypovirulence. Phytopathology 87:50-59.

Bryner, S. F., and Rigling, D. 2011. Temperature-dependent genotype-bygenotype interaction between a pathogenic fungus and its hyperparasitic virus. Am. Nat. 177:65-74

Dutech, C., Barrès, B., Bridier, J., Robin, C., Milgroom, M. G., and Ravigné, V. 2012. The chestnut blight fungus world tour: Successive introduction events from diverse origins in an invasive plant fungal pathogen. Mol. Ecol. 21:3931-3946.

Dutech, C., Fabreguettes, O., Capdevielle, X., and Robin, C. 2010. Multiple introductions of divergent genetic lineages in an invasive fungal pathogen, Cryphonectria parasitica, in France. Heredity 105:220-228.

Early, R., Bradley, B. A., Dukes, J. S., Lawler, J. J., Olden, J. D., Blumenthal, D. M., Gonzalez, P., Grosholz, E. D., Ibañez, I., Miller, L. P., Sorte, C. J., and Tatem, A. J. 2016. Global threats from invasive alien species in the twenty-first century and national response capacities. Nat. Commun. 7: Article 12485.

EFSA PLH Panel (EFSA Panel on Plant Health), Jeger, M., Bragard, C., Chatzivassiliou, E., Dehnen-Schmutz, K., Gilioli, G., Jaques Miret, J. A., MacLeod, A., Navajas Navarro, M., Niere, B., Parnell, S., Potting, R., Rafoss, T., Urek, G., Van Bruggen, A., Werf, W., West, J., Winter, S., Maresi, G., Prospero, S., Vettraino, A. M., Vloutoglou, I., Pautasso, M., and Rossi, V. 2016. Scientific opinion on the risk assessment and reduction options for Cryphonectria parasitica in the EU. EFSA J. 14(12):4641. doi: 10.2903/j.efsa.2016.4641

Ellison, A. M., Bank, M. S., Clinton, B. D., Colburn, E. A., Elliott, K., Ford, C. R., Foster, D. R., Kloeppel, B. D., Knoepp, J. D., Lovett, G. M., Mohan, J., Orwig, D. A., Rodenhouse, N. L., Sobczak, W. V., Stinson, K. A., Stone, J. K., Swan, C. M., Thompson, J., Von Holle, B., and Webster, J. R. 2005. Loss of foundation species: Consequences for the structure and dynamics of forested ecosystems. Front. Ecol. Environ. 3:479-486.

Eschen, R., Britton, K., Brockerhoff, E., Burgess, T., Dalley, V., Epanchin-Niell, R. S., Gupta, K., Hardy, G., Huang, Y., Kenis, M., Kimani, E., Li, H.-M., Olsen, S., Ormrod, R., Otieno, W., Sadof, C., Tadeu, E., and Theyse, M. 2015. International variation in phytosanitary legislation and regulations governing importation of plants for planting. Environ. Sci. Policy 51:228-237.

Gibbs, J. N. 1978. Intercontinental epidemiology of Dutch elm disease. Annu. Rev. Phytopathol. 16:287-307.

Gobbin, D., Hoegger, P. J., Heiniger, U., and Rigling D. 2003. Sequence variation and evolution of Cryphonectria hypovirus 1 (CHV-1) in Europe. Virus Res. 97:39-46.

Grente, J. 1965. Les formes hypovirulentes d'Endothia parasitica et les espoirs de lutte contre le chancre du chataignier. C. R. Acad. Agric. Fr. 51:1033-1037.

Gross, A., Holdenrieder, O., Pautasso, M., Queloz, V., and Sieber, T. N. 2014. Hymenoscyphus pseudoalbidus, the causal agent of European ash dieback. Mol. Plant Pathol. 15:5-21.

Heiniger, U., and Rigling, D. 1994. Biological control of chestnut blight in Europe. Annu. Rev. Phytopathol. 32:581-599.

Hoegger, P. J., Rigling, D., Holdenrieder, O., and Heiniger, U. 2000. Genetic structure of newly established populations of Cryphonectria parasitica. Mycol. Res. 104:1108-1116.

Hoegger, P. J., Rigling, D., Holdenrieder, O., and Heiniger, U. 2002. Cryphonectria radicalis: Rediscovery of a lost fungus. Mycologia 94:105-115.

Hohl, H. R., and Iselin, K. 1984. Strains of Phytophthora infestans from Switzerland with A2 mating type behaviour. Trans. Br. Mycol. Soc. 83:529-530.
Kamvar, Z. N., Tabima, J. F., and Grünwald, N. J. 2014. Poppr: An R package for genetic analysis of populations with clonal, partially clonal, and/or sexual reproduction. PeerJ 2:e281.

Kiiker, R., Hansen, M., Williams, I. H., Cooke, D. E. L., and Runno-Paurson, E. 2018. Outcome of sexual reproduction in the Phytophthora infestans population in Estonian potato fields. Eur. J. Plant Pathol. 152:395-407.

Leclerc, M., Doré, T., Gilligan, C. A., Lucas, P., and Filipe, J. A. N. 2014. Estimating the delay between host infection and disease (incubation period) and assessing its significance to the epidemiology of plant diseases. PLoS One 9:e86568.

Liu, Y.-C., and Milgroom, M. G. 2007. High diversity of vegetative compatibility types in Cryphonectria parasitica in Japan and China. Mycologia 99: 279-284

Loo, J. A. 2009. Ecological impacts of non-indigenous invasive fungi as forest pathogens. Pages 81-96 in: Ecological Impacts of Non-Native Invertebrates and Fungi on Terrestrial Ecosystems. D. W. Langor and J. Sweeney, eds. Springer Netherlands, Dordrecht, The Netherlands.

Maloy, O. C. 1997. White pine blister rust control in North America: A case history. Annu. Rev. Phytopathol. 35:87-109.

Mariette, N., Mabon, R., Corbière, R., Boulard, F., Glais, I., Marquer, B., Pasco, C., Montarry, J., and Andrivon, D. 2016. Phenotypic and genotypic changes in French populations of Phytophthora infestans: Are invasive clones the most aggressive? Plant Pathol. 65:577-586.

May, R. M., and Anderson, R. M. 1983. Epidemiology and genetics in the coevolution of parasites and hosts. Proc. R. Soc. Lond. B Biol. Sci. 219:281-313.

Milgroom, M. G., and Cortesi, P. 2004. Biological control of chestnut blight with hypovirulence: A critical analysis. Annu. Rev. Phytopathol. 42:311-338.

Montarry, J., Andrivon, D., Glais, I., Corbiere, R., Mialdea, G., and Delmotte, F. 2010. Microsatellite markers reveal two admixed genetic groups and an ongoing displacement within the French population of the invasive plant pathogen Phytophthora infestans. Mol. Ecol. 19:1965-1977.

Morais, P., and Reichard, M. 2018. Cryptic invasions: A review. Sci. Total Environ. 613-614:1438-1448.

Pažitný, J., Bolvanský, M., and Adamčíková, K. 2018. Screening for resistance of progenies derived from Castanea sativa $\times C$. crenata and $C$. crenata to Cryphonectria parasitica. For. Pathol. 48:e12439.

Philibert, A., Desprez-Loustau, M.-L., Fabre, B., Frey, P., Halkett, F., Husson, C., Lung-Escarmant, B., Marçais, B., Robin, C., Vacher, C., and Makowski, D. 2011. Predicting invasion success of forest pathogenic fungi from species traits. J. Appl. Ecol. 48:1381-1390.

Picard, C., Afonso, T., Benko-Beloglavec, A., Karadjova, O., Matthews-Berry, S., Paunovic, S. A., Pietsch, M., Reed, P., van der Gaag, D. J., and Ward, M. 2018. Recommended regulated non-quarantine pests (RNQPs), associated thresholds and risk management measures in the European and Mediterranean region. EPPO Bull. 48:552-568.

Prospero, S., and Rigling, D. 2012. Invasion genetics of the chestnut blight fungus Cryphonectria parasitica in Switzerland. Phytopathology 102:73-82.

R Core Team. 2014. R: A Language and Environment for Statistical Computing. R Foundation for Statistical Computing, Vienna, Austria.

Rigling, D., and Prospero, S. 2018. Cryphonectria parasitica, the causal agent of chestnut blight: Invasion history, population biology and disease control. Mol. Plant Pathol. 19:7-20.

Robin, C., Andanson, A., Saint-Jean, G., Fabreguettes, O., and Dutech, C. 2017. What was old is new again: Thermal adaptation within clonal lineages during range expansion in a fungal pathogen. Mol. Ecol. 26:1952-1963.

Robin, C., and Heiniger, U. 2001. Chestnut blight in Europe: Diversity of Cryphonectria parasitica, hypovirulence and biocontrol. For. Snow Landsc. Res. 76:361-367.

Sacristán, S., and García-Arenal, F. 2008. The evolution of virulence and pathogenicity in plant pathogen populations. Mol. Plant Pathol. 9:369-384.

Schoebel, C. N., Botella, L., Lygis, V., and Rigling, D. 2017. Population genetic analysis of a parasitic mycovirus to infer the invasion history of its fungal host. Mol. Ecol. 26:2482-2497.

Schrader, G., and Unger, J.-G. 2003. Plant quarantine as a measure against invasive alien species: The framework of the international plant protection convention and the plant health regulations in the European Union. Biol. Invasions 5:357-364.

Shackleton, R. T., Biggs, R., Richardson, D. M., and Larson, B. M. H. 2018. Social-ecological drivers and impacts of invasion-related regime shifts: Consequences for ecosystem services and human wellbeing. Environ. Sci. Policy 89:300-314.

Wuest, C. E., Harrington, T. C., Fraedrich, S. W., Yun, H.-Y., and Lu, S.-S. 2017. Genetic variation in native populations of the laurel wilt pathogen, Raffaelea lauricola, in Taiwan and Japan and the introduced population in the United States. Plant Dis. 101:619-628.

Xenopoulos, S. G., and Papachatzis, A. 1999. Problems of chestnut growing in Greece: Screening for resistance of several chestnut provenances to Cryphonectria (Endothia) parasitica (Murr.) Anderson. Acta Hortic. 494:521-528. 